\title{
Basal electrical activity in the anal canal in man
}

\author{
W. J. WANKLiNG, B. H. BROWN, C. D. COLlins, AND H. L. DUThiE \\ From the University Department of Surgery, The Royal Infirmary, Sheffield
}

Electrical activity in the region of the anal canal in man has been previously recorded on many occasions from the voluntary muscle of the external anal sphincter. We have attempted in addition to delineate the electrical activity arising in the smooth muscle surrounding the anal canal with the aim of assessing the relative roles of the two components in preserving anal continence. A preliminary report of some of the data was made to the Surgical Research Society in July 1967 (Wankling, Brown, and Duthie, 1968).

\section{METHOD}

Studies were made on 20 men aged between 20 and 55 years who had no anorectal disease. Four parameters were continuously monitored and recorded on a $U / V$ recorder: (1) electrical activity in the anal canal, (2) radial force in the anal canal, (3) respiration, and (4) external anal sphincter activity.

The electrical activity was recorded from two stainless steel bands $5 \mathrm{~mm}$ wide mounted coaxially, $1 \mathrm{~cm}$ apart, on a $1 \mathrm{~cm}$ diameter Perspex rod which was inserted into the anal canal (Fig. 1). The signals detected were amplified, using an $\mathrm{A} / \mathrm{C}$ amplifier with a frequency response within $\pm 3 \mathrm{db}$ from $0.02 \mathrm{~Hz}$ to $1 \mathrm{k} \mathrm{Hz}$, and displayed on the recorder at a sensitivity of $200 \mu \mathrm{v} / \mathrm{cm}$. The extremely low frequency response of this amplifier was necessary in order to record very slow activity and yet be unaffected by direct voltages at the surface of the recording electrodes.

The transducer to measure radial force consisted of a steel cantilever, incorporating a semiconductor strain gauge, mounted between the two stainless steel electrodes (Fig. 1). The strain gauge was placed in one arm of a resistance bridge, the output of which was amplified and displayed on the recorder at a sensitivity corresponding to $1 \mathrm{grm} / \mathrm{cm}$. The cantilever was only sensitive to radial force between the electrical recording electrodes and was not affected by forces parallel to the axis of the rod. With the aid of a mark on the side of the shaft, the obturator was placed in the anal canal so that the cantilever was $2.5 \mathrm{~cm}$ from the anal verge and the centres of the electrodes $1.75 \mathrm{~cm}$ and $3.25 \mathrm{~cm}$ respectively from the anal verge. The obturator was held in position by a length of adhesive tape lightly attached to the buttocks. Little or no movement of the obturator was noted during the tests and certainly no regular alteration in position which could have occasioned the wave forms observed. We have chosen to measure radial force in the anal canal as an index of muscle activity, as our previous experience has suggested that it may be a more stable parameter than intraluminal pressure in this region (Collins, Duthie, Shelley, and Whittaker, 1967).

Respiration was monitored as a means of distinguishing true wave activity in the electrical and force records from artefacts produced by respiration. A pneumograph placed round the patient's chest and connected to a pressure transducer was used for this purpose.

The activity of the external anal sphincter was recorded using a concentric needle electrode placed into the muscle. This was found preferable to the use of surface electrodes which were difficult to attach and very much less sensitive than the needle electrodes, which enabled resting tone to be detected even in the anaesthetized patients.

Each man had three recording sessions: the first at rest on his left side, the second after induction of light general anaesthesia, and the third when complete muscle relaxation was added to the light anaesthesia. The last two sessions were restricted to 10 to $15 \mathrm{~min}$ duration. The complete disappearance of the electromyogram from the external anal sphincter was taken as indicating full muscular relaxation. Premedication was with morphine, $13 \mathrm{mg}$, atropine, $0.6 \mathrm{mg}$. Anaesthesia was induced with a sleep dose of thiopentone, $2.5 \%$, with maintenance nitrous oxide, oxygen, and halothane, and muscle relaxation with gallamine triethoxide.

\section{RESULTS}

BASAL ELECTRICAL SLOW WAVE ACTIVITY In 52 of the 60 records on the 20 subjects a wave form was observed with a frequency of 10 to 20 per min and

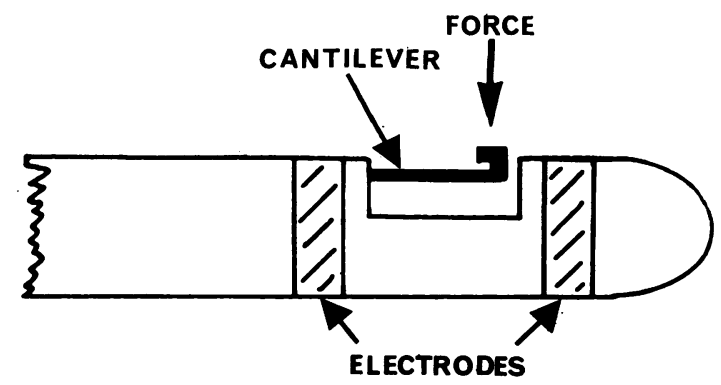

FIG. 1. Diagram of tip of anal canal obturator showing the disposition of the electrodes and the cantilever for force measurement. 
an amplitude of 50 to 400 microvolts (Fig. 2). It was seen in all subjects on one or more record and was called the 'slow wave' or 'basal electrical activity'. In the conscious subjects the mean frequency was 16.4 per min and the mean amplitude was 153 microvolts. Neither the induction of anaesthesia nor the complete paralysis of the external anal sphincter significantly altered these values (Table I). It was not coincident with respiratory movements. The frequency was steady but the amplitude was not constant in each subject. Phasic increases and decreases were observed, especially when ultraslow wave activity (vide infra) was present. In these circumstances the amplitude of the basal electrical activity was greatest at the peak of the slow waves. No spikes or bursts of fast activity were distinguished in these records.

ULTRASLOW WAVE ELECTRICAL ACTIVITY In 36 of the 60 records on the 20 subjects a slower form of electrical activity was observed. This 'ultraslow wave' activity consisted of a long slow change in the base line during a period in which the basal electrical activity was being recorded (Fig. 3) and was seen in 14 subjects. It was sufficiently regular to allow measurement of the frequency in only 16 records. The frequency ranged from 1 to 2 per min with an amplitude of 200 to 2,000 microvolts. Neitherl the induction of anaesthesia nor the complete paraysis of the external anal sphincter altered significantly these values (Table I).

SLOW FORCE WAVES IN THE ANAL CANAL In 52 of the 60 records from 20 subjects a wave form was observed with a frequency of 10 to 20 per min and an amplitude of 0.2 to $1.0 \mathrm{~g}$ wt (Fig. 2). These waves were seen at some time in all 20 subjects and were called 'slow waves'. In the conscious subjects they had a mean frequency of 16.9 per min and a mean amplitude of $0.6 \mathrm{~g}$ weight. Neither the induction of anaesthesia nor the complete paralysis of the external anal sphincter altered significantly these values (Table II). The same phasic increase in amplitude as seen in the basal electrical activity was observed in the slow force waves when they were present together with ultraslow force waves (vide infra).

ULTRASLOW FORCE WAVES IN THE ANAL CANAL As in the electrical activity, these ultraslow waves were seen in a smaller proportion of the recordings ( 26 of 60 records) than the slow force waves. They were seen in 14 of the 20 subjects (Table II). On a few occasions ultraslow force waves were present in the absence of slow force waves (Fig. 3), but on the whole the two wave forms occurred together.

CORRELATION BETWEEN ELECTRICAL ACTIVITY AND FORCE The similarity in frequency of the two forms

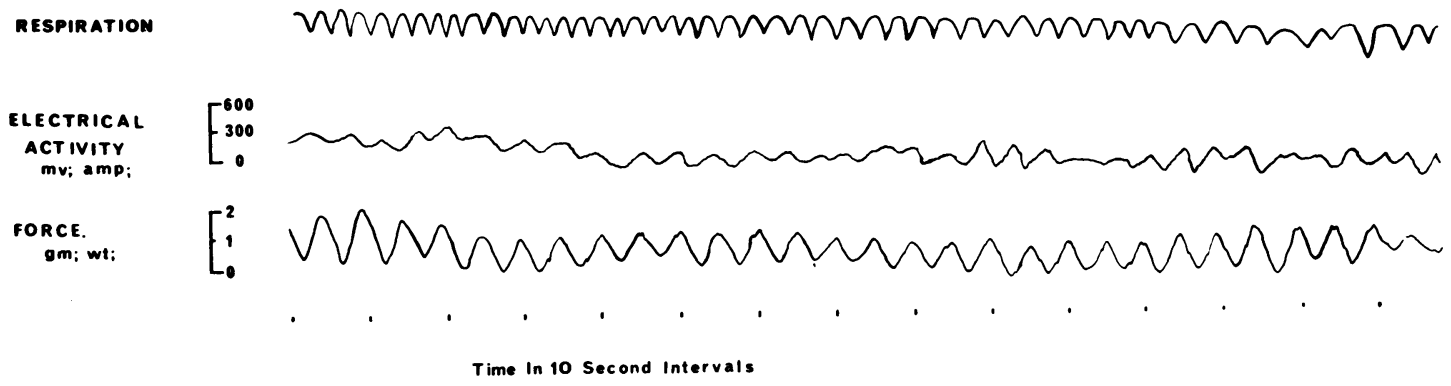

FIG. 2. Tracing showing slow wave activity on both electrical and force recordings.

TABLE I

CHARACTERISTICS OF ELECTRICAL WAVE ACTIVITY IN THE ANAL CANAL

Electrical Waves $^{1}$

Awake Anaesthetized Paralysed

Slow Waves

Frequency per min.

Amplitude (microvolts

Awake

Ultraslow Waves

Frequency per min

Amplitude (microvolts)

$16.4 \pm 0.9$
$153 \pm 21$

$15 \cdot 3 \pm 0 \cdot 8$
$164 \pm 12$
$1 \cdot 2 \pm 0 \cdot 2$
$838 \quad(19)$
\pm 100

$14 \cdot 6 \pm 0.8$

$142 \pm 24$ (16)

$1 \cdot 4 \pm 0.2 \quad(5)$

$500 \begin{array}{ccc}1 \cdot 4 & \pm 0 \cdot 2 & (5) \\ & \pm 93 & (5)\end{array}$

1 Mean \pm standard error of mean.

Numbers in brackets indicate number of subjects out of 20 showing wave form. 
TABLE II

CHARACTERISTICS OF FORCE WAVE ACTIVITY IN THE ANAL CANAL

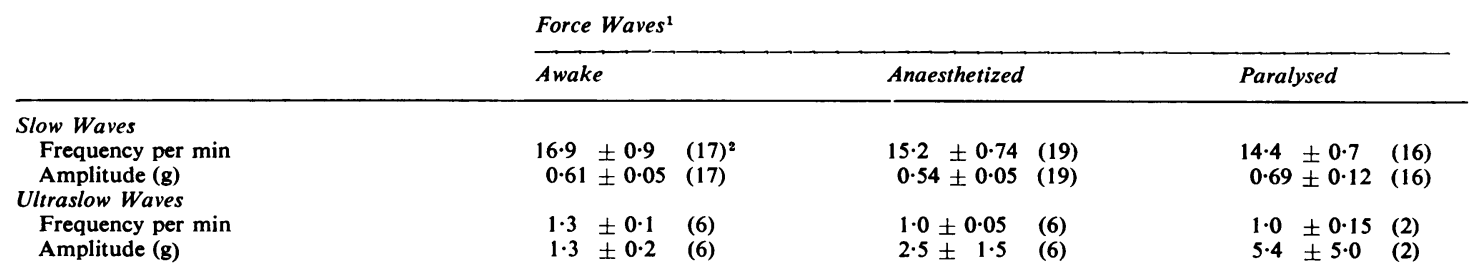

${ }^{1}$ Mean \pm standard error of mean.

2 Numbers in brackets indicate number of subjects out of 20 showing wave form.

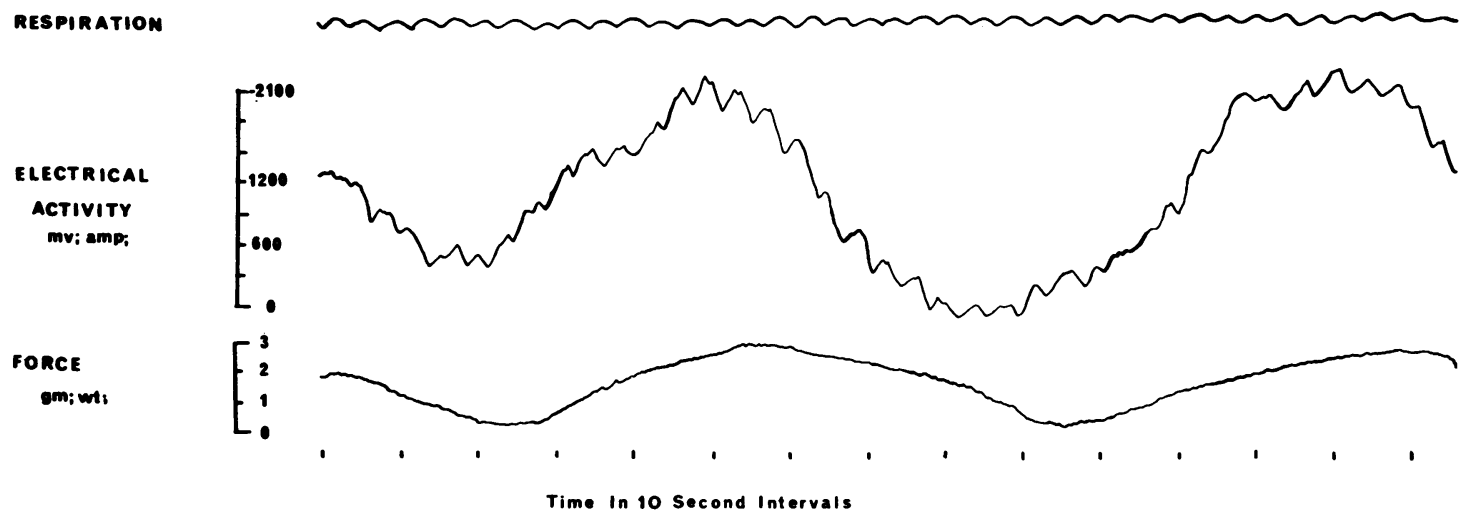

FIG. 3. Tracing showing ultraslow wave activity on both electrical and force recordings.

of activity naturally led to attempts to correlate them. While, on many occasions the electrical and force slow waves coincided for a few cycles, no general relationship could be established.

\section{DISCUSSION}

The aim of this study was to record electrical activity from the smooth muscle component of the anal sphincters in man. The situation is more complex than in other parts of the alimentary canal from which electrical activity has been measured because of the presence of the voluntary muscle fibres of the external anal sphincter and of the pelvic floor which surround the smooth muscle in this region. Furthermore these voluntary muscle fibres exhibit a continuous spontaneous activity. We suggest that the wave forms we have observed emanate from smooth muscle because the wave forms were recorded when the voluntary muscles were paralysed, and the form and frequency of the waves were similar to those previously recorded from the small bowel in man (Daniel, Wachter, Honour, and Bagoch, 1960). Although the frequency was of the same order, at a mean of about 16 per min, it was higher than the mean frequency of nearly 12 per min observed in the duodenum of man using bipolar intraluminal electrodes (Christensen, Clifton, and Schedl, 1966) and than the 7 per min observed in the ileum in man (Daniel et al, 1960). This would suggest that the colon and rectum may have a rhythm of their own and are not influenced by the gradient which exists in the small intestine, where, in both man and dog, a more rapid rate is found in the duodenum than in the rest of the small gut with a gradual decrease in frequency along the jejunum and ileum. In confirmation of this idea it has been shown that the electrical activity of the terminal ileum does not pass across the ileocaecal valve in dogs (Daniel and Chapman, 1963).

The basal electrical activity of the colon and rectum in man has not been reported so there is no evidence to establish whether or not a gradient exists along the large bowel. Extensive work by Bülbring's group on the intracellular measurement of electrical activity in the smooth muscle of the taenia coli of the guinea pig has given insight into the basic problems of smooth muscle activity (eg Bülbring and Kuriyama, 1963) but extracellular measurements in animals are few in number. In a 
study on the sigmoid colon in man, Tsuchida and Kimura (1966), using bipolar electrodes, did not distinguish a basal electrical rhythm but were able to record fast 'spike' potentials which occurred in bursts lasting about $2 \mathrm{sec}$ with intervals of just under a minute between bursts. Both slow waves and fast activity have been shown on the studies in the small bowel in dogs and man. However, we have only identified slow waves in the anal canal. This latter finding is all the more surprising because localization of the slow or basal electrical activity in the small bowel has shown it to be in the longitudinal muscle coat (Daniel and Chapman, 1963) and the bulk of the smooth muscle around the anal canal is circular in the internal anal sphincter.

There has been much discussion on the significance of the basal electrical activity of the intestine (Daniel and Chapman, 1963). It is thought to provide a background electrical potential which permits depolarization of the individual fibres at the time of their contraction. This shows as the fast or 'spike' electrical activity which has been found to be associated with the motility of the small intestine. The wave forms seen on the force recording from the anal canal in the present study had an almost identical frequency to the electrical activity. However, we are not yet satisfied that they are causally connected. Further study of the progression of these waves along the bowel may help to elucidate the problem.

SUMMARY

Electrical activity in the anal canal has been studied in 20 subjects using bipolar intraluminal electrodes and simultaneously radial force in the anal canal was measured. Slow or basal electrical activity was recorded in all subjects at a mean frequency of 16.4 per min with the subjects resting on the left side. An ultraslow wave form was noted in 14 of the 20 subjects at a mean frequency of 1.6 per min. A similar pattern of waves was shown on the force recording-slow 16.9 per min and ultraslow 1.3 per min. The electrical and force wave activities were not always in phase. Paralysis of the external anal sphincter did not significantly alter the characteristics of electrical or force waves.

We acknowledge with thanks the assistance of the Sheffield Regional Medical Physics Department.

\section{REFERENCES}

Bülbring, E., and Kuriyama, H. (1963). Effects of changes in the external sodium and calcium concentrations on spontaneous electrical activity in smooth muscle of guinea-pig taenia coli. J. Physio. (Lond.), 166, 29-58.

Christensen, J., Clifton, J. A., and Schedl, H. P. (1966). Variations in the frequency of the human duodenal basic electrical rhythm in health and disease. Gastroenterology, 51, 200-206.

Collins, C. D., Duthie, H. L., Shelley, T., and Whittaker, G. E. (1967). Force in the anal canal and anal continence. Gut, 8, 354-360.

Daniel, E. E., and Chapman, K. M. (1963). Electrical activity of the gastrointestinal tract as an indication of mechanical activity. Amer. J. dig. Dis., 8, 54-102.

—_, Wachter, B. T., Honour, A. J., and Bagoch, A. (1960). The relationship between electrical and mechanical activity of the small intestine of $\mathrm{dog}$ and man. Canad. J. Biochem., 38, 777-802

Tsuchida, S., and Kimura, Y. (1966). Electromyography of the intestines by the intra-intestinal method. Tohoku J. exp. Med., 89, 61-68.

Wankling, W. J., Brown, B. H., and Duthie, H. L. (1968). Basal electrical activity of the anal canal. (Abstract.) Brit. J. Surg., $55,158$. 